\title{
Diffusion-Related SOFC Stack Degradation
}

\author{
Norbert H. Menzler ${ }^{1}$, Alexander Beez ${ }^{1,2}$, Nikolas Grünwald ${ }^{1}$, Doris Sebold ${ }^{1}$, Qingping \\ Fang $^{1}$, Robert Vaßen ${ }^{1}$ \\ ${ }^{1}$ Institute of Energy and Climate Research (IEK), Forschungszentrum Jülich GmbH, \\ 52425 Jülich, Germany \\ ${ }^{2}$ Christian-Doppler-Laboratory for Interfaces in Metal-Supported Electrochemical Energy \\ Converters, 52425 Jülich, Germany
}

\begin{abstract}
As part of two different stack tests with four-plane short stacks and their intensive post-test characterization, two varying diffusionrelated degradation mechanisms were investigated. The first was a short-term test $(\sim 1250 \mathrm{~h})$ with two different chromium evaporation protection layers on the air-side metallic interconnect and frame and the second was a long-term endurance test $(\sim 35,000 \mathrm{~h})$. For the first stack, two planes were coated with a manganese oxide layer applied by wet powder spraying (WPS), while the other two planes were coated with a manganese-cobalt-iron spinel layer by atmospheric plasma spraying (APS). The voltage loss in the planes with a WPScoated interconnect was markedly higher than in those coated by means of APS. Finally, it was shown that the microstructure of the layers plays a key role in minimizing $\mathrm{Cr}$ evaporation. In this stack, gas-phase diffusion prevails over degradation. In the long-term stack, severe degradation due to solid-state manganese diffusion was observed. This paper draws an interaction hypothesis.
\end{abstract}

\section{Introduction}

Solid oxide fuel cell systems for stationary applications should exhibit low degradation $(<<$ $1 \% / 1,000 \mathrm{~h}$ voltage loss) to ensure long-term operation (> 40,000h). The relatively high operation temperature of SOFCs $\left(\geq 700^{\circ} \mathrm{C}\right)$ is, on the one hand, beneficial for oxygen conduction through the ceramic-based electrolyte, but, on the other hand, all diffusionrelated, energetically driven mechanisms are also facilitated. This is less marked for gasdiffusion processes but more pronounced for solid-state diffusion (surface, bulk, grain boundary). For gas-diffusion processes, other parameters such as gas stream, individual partial pressures, and partial pressure gradients are dominant.

As part of two different stack tests with four-plane short stacks and their intensive post-test characterization, two varying diffusion-related degradation mechanisms were investigated. The first was a short-term test $(\sim 1250 \mathrm{~h})$ with two different chromium evaporation protection layers on the air-side metallic interconnect and frame and the second was a longterm endurance test $(\sim 35,000 \mathrm{~h})$. For the first stack, two planes were coated with a manganese oxide layer applied by wet powder spraying (WPS), while the other two planes were coated with a manganese-cobalt-iron spinel layer by atmospheric plasma spraying (APS). The protective layers thus not only differ in terms of coating technology but also with respect to material. The voltage loss in the planes with a WPS-coated interconnect was markedly higher than in those coated by means of APS. Finally, it was shown that the 
microstructure of the layers plays a key role in minimizing Cr evaporation. In this stack, gas-phase diffusion prevails over degradation.

In the stack in long-term operation, the influence of an element diffusing from the cathodic contact layer towards and through the electrolyte was characterized. Manganese, which probably originates from the contact layer (but is also part of the interconnect protection layer), diffuses via solid-state diffusion and reacts with the zirconia-based electrolyte while reaching reducing conditions in the fuel compartment. This interaction forms a secondary sponge-like porous phase at the electrolyte-anode boundary. This secondary phase causes stresses and - after growing to relatively large islands of around ten micrometers in diameter - leads to electrolyte-anode delamination and finally to complete cell failure. For the Mn-related degradation, it is assumed that solid-state diffusion occurs, as the electrolyte is coated by a dense thin-film diffusion barrier layer which suppresses gas diffusion.

Both results indicate that volatile elements (which could either evaporate via the gas phase or diffuse via the solid state) should be avoided as they can lead to severe cell, stack, and system failure - even after thousands of hours of high-temperature operation.

\section{Stack description}

Two different Jülich F-design stacks were used for the investigations. Both stacks are composed of four planes with standard Jülich anode-supported solid oxide fuel cells that are $100 \times 100 \mathrm{~mm}^{2}$ in size and have an active area of $80 \mathrm{~cm}^{2}$. The substrate and anode are a mixture of $\mathrm{Ni}(\mathrm{O})$ with yttria-stabilized zirconia (8mol\%, 8YSZ), the electrolyte is pure 8YSZ, the diffusion barrier layer is based on gadonilia-doped ceria (GDC), and the cathode is an A-site-deficient $\mathrm{La}-\mathrm{Sr}-\mathrm{Fe}-\mathrm{Co}$ (LSCF) perovskite (1). One of the stacks (F 1004-21), operating for nearly $35,000 \mathrm{~h}$, has a GDC layer applied by physical vapor deposition (d $1 \mu \mathrm{m})$ and the other (F 1004-69) has a screen-printed and sintered layer (d $\sim 5 \mu \mathrm{m})$. Both stacks have interconnects based on Crofer 22 APU, a glass-ceramic sealant, and a Ni mesh as the anodic contact. In the $\mathrm{F}$ 1004-21 stack, the $\mathrm{Cr}$ retention layer was applied by atmospheric plasma spraying (APS) and is composed of a Mn-Co-Fe spinel (MCF) $(2,3)$; the cathodic contact is ensured by a wet powder-sprayed (WPS) perovskite contact layer (La-Mn-Cu-Co; LCC12). In the F 1004-69 stack, LSCF was used as a contact material and two different $\mathrm{Cr}$ retention layers were applied. In two of the planes, a similar layer to one found in F 1004-21 was used and in the other two planes, the older WPS Mn oxide layer was used as a retention coating (4).

The F $1004-21$ stack operated for $34,500 \mathrm{~h}$ at $700^{\circ} \mathrm{C}$ and $0.5 \mathrm{~A} / \mathrm{cm}^{2}$ with a fuel utilization of $40 \%$, while the F $1004-69$ stack operated for only $1,250 \mathrm{~h}$ at $700^{\circ} \mathrm{C}$. For the first $240 \mathrm{~h}$, a current density of $0.5 \mathrm{~A} / \mathrm{cm}^{2}$ was chosen and subsequently raised to $0.75 \mathrm{~A} / \mathrm{cm}^{2}$. The idea behind this was to change the overpotential at the interfaces and especially to clarify whether this influences the interaction of LSCF with volatile $\mathrm{Cr}$ species. This was performed based on the results of a thin-film electrolyte and barrier layer stack which showed $\mathrm{SrCrO}_{4}$ formation for the first time not just on the top of the cathode layer -as is usually observed - but also at the cathode-barrier layer interface (ion conductor) (5). The stack in long-term operation was shut down due to the progressive voltage degradation of one layer, starting at $\sim 28,000 \mathrm{~h}$. 


\section{Results of post-test analysis}

\section{$\underline{\mathrm{Cr} \text { amount in LSCF }}$}

The amount of $\mathrm{Cr}$ incorporated into the LSCF cathode after the individual operation time was measured by leaching the cathode from the cell and subsequently by quantitative analysis of the solution by means of ICP-OES* (for more details please refer to (6)). The amounts of $\mathrm{Cr}$ per $\mathrm{cm}^{2}$ were less than $5 \mu \mathrm{g}$ for the two layers with APS coating in the F

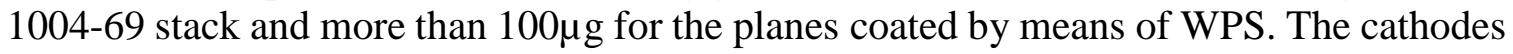
of the stack in long-term operation show very low chromium amounts of approx. $5 \mu \mathrm{g}-$ even after more than $30,000 \mathrm{~h}$ of operation.

\section{$\underline{\text { Microstructural investigations }}$}

Cross-sectional SEM micrographs of the two stacks are depicted in Figures 1 to 3.

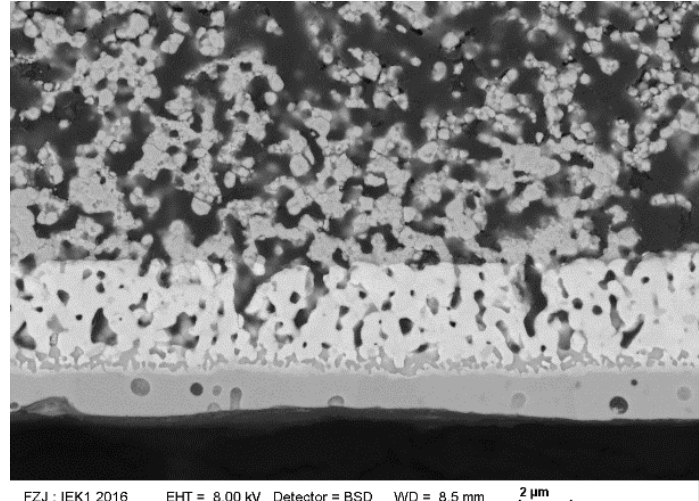

FZJ : IEK1 $2016 \quad E H T=8.00 \mathrm{kV}$ Detector $=\mathrm{BSD} \quad \mathrm{WD}=8.5 \mathrm{~mm} \stackrel{2 \mu \mathrm{mm}}{\longmapsto}$

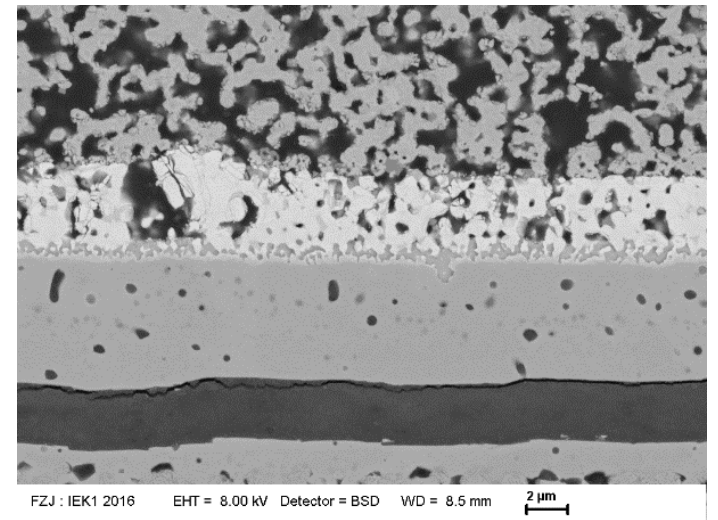

Figure 1. SEM cross sections of cells from the F 1004-69 stack. Left: electrolyte-barriercathode interface from a plane with APS coating; right: electrolyte-barrier-cathode interface from a plane with WPS coating (the cracks are due to sample preparation; cathode on the top)
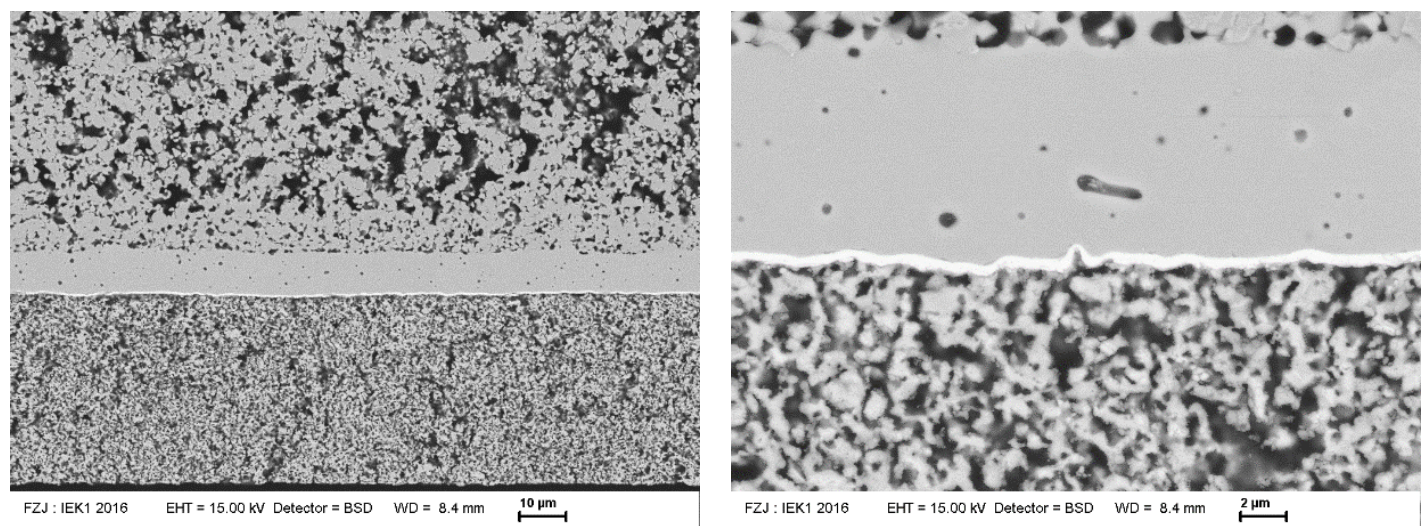

Figure 2. SEM cross section of the cell of a non-degraded plane of the F 1004-21 stack after $34,500 \mathrm{~h}$ of operation (note that in this figure the cathode side is on the bottom) 

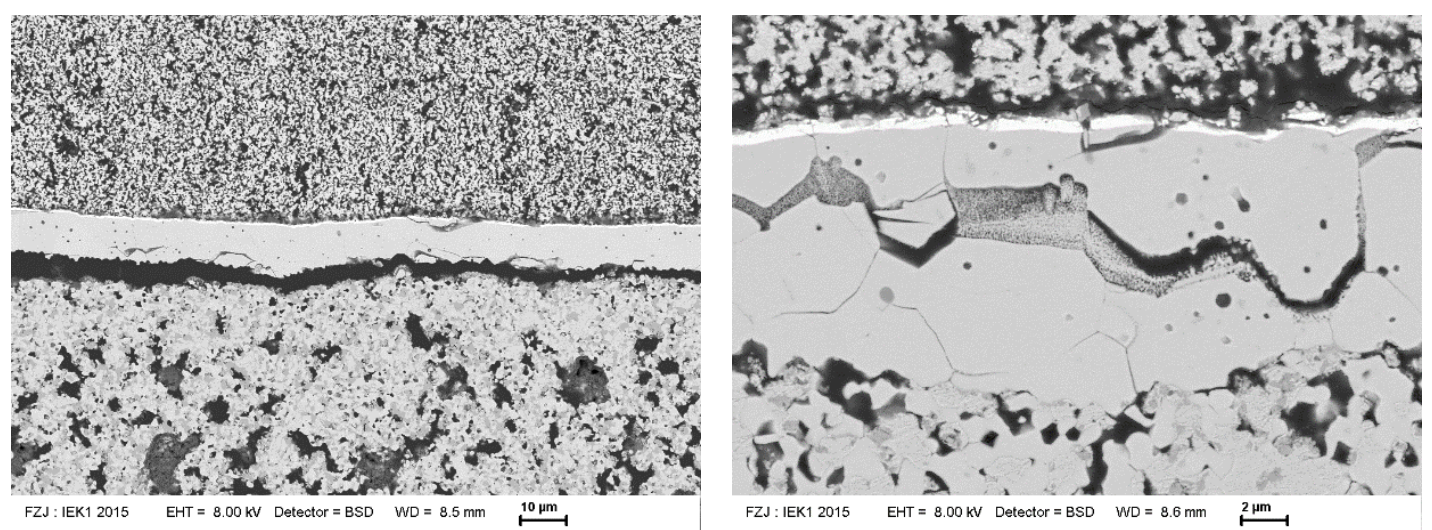

Figure 3. SEM cross section of the progressive degraded cell of the F 1004-21 stack (cathode on top)

In the micrographs from Figure 1, two microstructural findings are evident. Firstly, at the LSCF/GDC interface of the cell with a WPS Mn oxide coating on the interconnect, small gray areas of novel phase formation are visible. EDX point analysis reveals the existence of $\mathrm{Cr}$ in these regions. Here, a $\mathrm{SrCrO}_{4}$-spinel has probably formed (due to the small size of the foreign phase the surrounding area was also analyzed). Secondly, the LSCF cathode appears somewhat fragmented, which means that the particles seem to be fractioned and partly detached from the main grains. This could be a secondary effect of the foreign phase formation. Cathodes with Sr contained in the lattice typically exhibit a SrO-containing surface region (7), which subsequently reacts with the volatile $\mathrm{Cr}$ species. The fractioning of the cathode could be caused by the strontium diffusing outwards and subsequently altering the physical characteristics of the remaining LSCF cathodes.

In Figure 2, one cell from the F 1004-21 stack which does not display progressive degradation is shown. There are no noticeable microstructural changes or secondary phases evident in any of the layers, electrolyte, barrier, cathode, and anode shown. The barrier layer applied by PVD uniformly covers the electrolyte surface with all its waviness and roughness.

In contrast, severe electrolyte deterioration and the complete detachment of the layer package - electrolyte-barrier-cathode - from the entire cell was observed in the progressively degraded plane (cf. Fig. 3). Additionally, within the electrolyte - especially along the grain boundaries - a fluffy, porous secondary phase was formed. EDX point analysis repeatedly shows zirconium, yttrium, oxygen, and manganese. Scandium, nickel, and other elements from the different layers were also found at random intervals. In regions exhibiting drastic delamination, large islands similar to the above-mentioned foreign phase formed facing the anode and growing from the electrolyte's fuel side surface (Figure 4). 


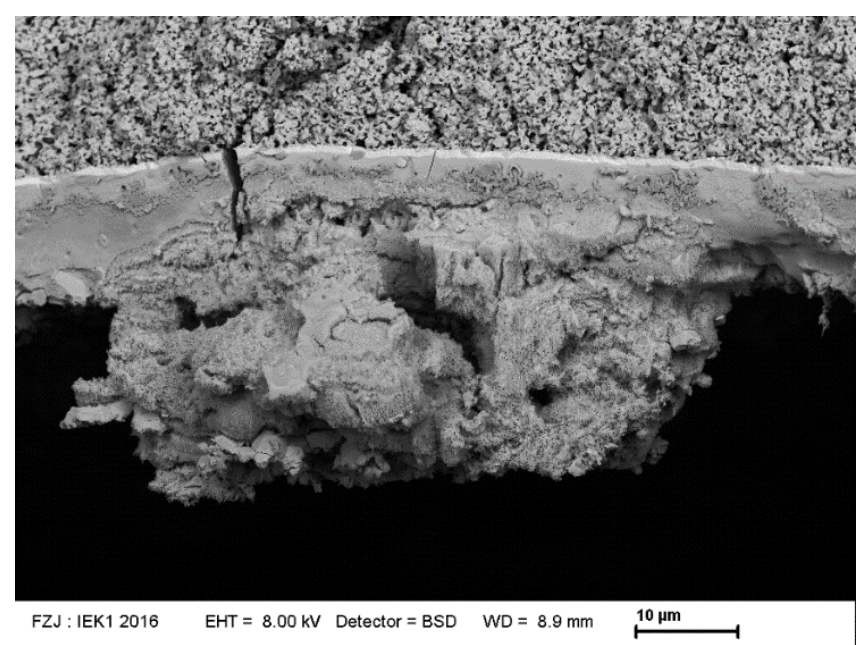

Figure 4. SEM micrograph of the progressively degraded cell of the F 1004-21 stack, exhibiting growth of porous islands originating from the electrolyte's surface towards the anode

\section{Discussion}

\section{$\underline{\text { F 1004-69 stack }}$}

A comparison of the two different types of $\mathrm{Cr}$ retention layers and the $\mathrm{Cr}$ species interaction with the LSCF cathode leads to the following conclusions:

- The MCF layer applied by APS acts as a powerful Cr retention layer; only trace amounts of $\mathrm{Cr}$ were measured.

- The manganese oxide layer coated by WPS was unable to hinder $\mathrm{Cr}$ from evaporating from the oxide scale surface interconnects; amounts of more than $100 \mu \mathrm{g} / \mathrm{cm}^{2}$ of cathode area were detected after short-term operation.

- In the cathode of the planes where the interconnect is coated by means of APS, no Cr was detected by SEM - neither at the cathode surface nor at any other location within the cell.

- The cathode of the cells within the WPS-coated interconnects is covered with $\mathrm{SrCrO}_{4}$ at random intervals (as published in the literature), but $\mathrm{SrCrO}_{4}$ was also found at the cathode-ion conductor boundary. This is unusual and unexpected but was observed once in a thin-film electrolyte stack. Here, the cathode microstructure also seems to be affected and the grains fragmented.

- High current densities $\left(>0.5 \mathrm{~A} / \mathrm{cm}^{2}\right)$ seem to change the underlying mechanisms which govern the interaction of $\mathrm{SrO}$, formed at the cathode grain surface, and the volatile $\mathrm{Cr}$ species

More details on this stack can be found in (8).

\section{$\underline{\text { F 1004-21 stack }}$}

This stack showed progressive degradation within one plane, starting at an operation time of $\sim 28,000 \mathrm{~h}$ and - to preserve possible findings and draw comparisons between the degrading plane and the other three - was shut down after 34,500h. The cells of the three 
non-degraded planes show typical microstructures for the single layers and evidently appear to be unchanged.

However, the degraded layer reveals severe electrolyte damage due to its interaction with manganese under reducing conditions. A secondary phase composed of $\mathrm{Zr}, \mathrm{Y}, \mathrm{O}$, and Mn was formed and its microstructure is highly porous. This secondary phase leads to stresses at the electrolyte-anode boundary and finally to delamination and cell cracking, which accounts for the progressive degradation of this plane. Intensive post-test SEM analysis of the other three cells reveals the possible start of a similar interaction in one plane. This phenomenon correlated with Mn was clearly observed (Figure 5), which means that the Mn diffusion and the subsequent interaction always occurs - but at random intervals. It is possible that the other planes would have exhibited similar behavior some hundreds or thousands of hours later. Figure 5 also shows that the grain surfaces facing a pore are covered with Mn oxide and no other element apart from oxygen was detected by EDX.

One possible explanation for this finding is that if a relatively high $\mathrm{Cr}$ vapor pressure exists (e.g. when using a WPS-coated interconnect), manganese originating from the contact layer reacts with chromium, thus forming $\mathrm{Cr}-\mathrm{Mn}$ spinel. If the $\mathrm{Cr}$ partial pressure is low (due to a well-functioning retention layer), the manganese has no reaction partner, diffuses towards the concentration gradient (high at a region near the interconnect due to MCF layer, low at the ion conductor region), and reaches the GDC barrier layer. The latter is efficient for $\mathrm{Sr}$ retention but evidently not for $\mathrm{Mn}$ retention. This might be explained by the vapor pressures of the underlying oxides. At $700^{\circ} \mathrm{C}$, the vapor pressure of $\operatorname{Sr}(\mathrm{OH})_{2}$ above $\mathrm{SrO}$ is $8.3 \times 10^{-10}$ bar $(\mathrm{SrO}$ activity $=1)$. Above chromia, the $\mathrm{CrO}_{3}$ or $\mathrm{Cr}(\mathrm{OH})_{2} \mathrm{O}_{2}$ pressure is under the same conditions of $5.7 \times 10^{-11}$ or $2.3 \times 10^{-8}$ bar, respectively. However, the vapor pressure of $\mathrm{MnO}_{2}$, which is the most likely compound to be formed in these conditions, is only $1 \times 10^{-19}$ bar, at least eight orders of magnitude less than, for example, chromium oxide/hydroxide and eleven orders of magnitude lower than $\mathrm{Sr}$ hydroxide. This means that manganese oxide does not form any volatile species under SOFC cathode conditions, but chromium and strontium oxide do. Dense GDC is therefore a good diffusion barrier for volatile Sr species but not for non-volatile manganese oxide diffused by surface or grain boundary diffusion. The Mn oxide can subsequently pass the barrier layer, then diffuse through the electrolyte via grain boundary diffusion, and react with the surrounding YSZ under reducing conditions. This leads to the formation of the sponge-like, porous secondary phase and finally to cell deterioration.
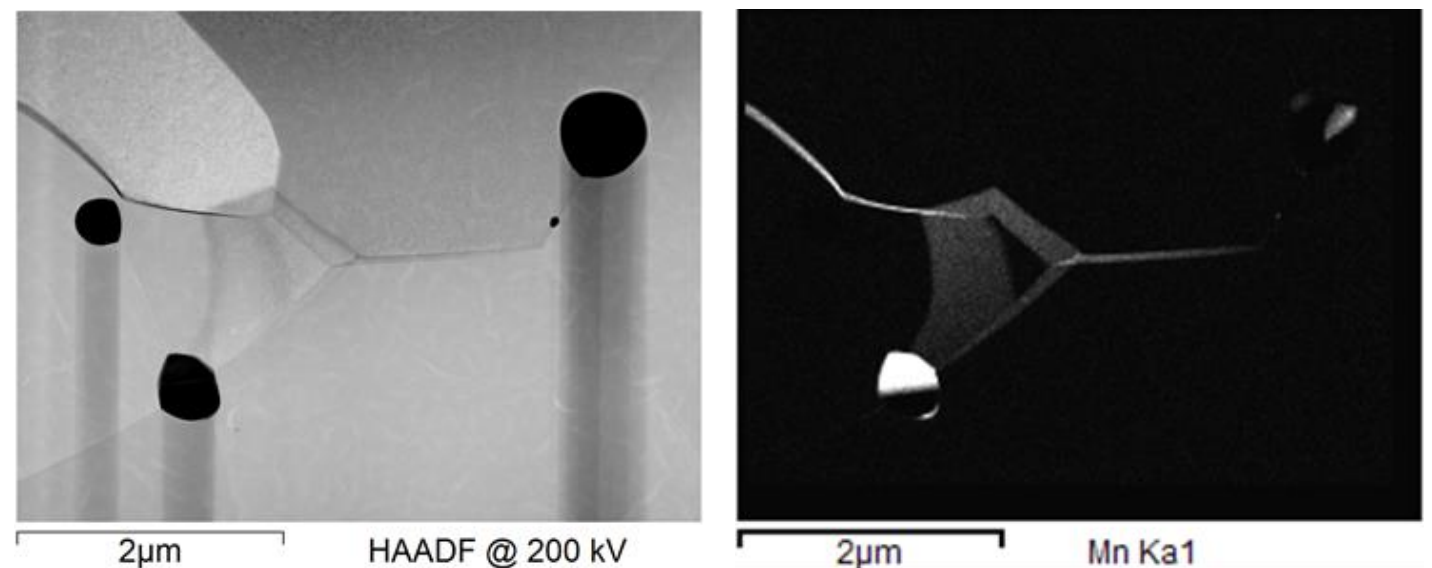

Figure 5. SEM (HAADF) cross section of grains and grain boundaries. Left: optical photo, right: EDX area-scan mapping of manganese 


\section{Conclusions}

Intensive post-test analysis of two different stacks with varied $\mathrm{Cr}$ retention layers and operated for different durations show that the interaction of materials based on diffusion possibly results in severe SOFC stack degradation. Some oxides are prone to evaporation under SOFC cathodic conditions, such as chromia or Sr oxide. They form volatile species which can then interact with other layers, scales, or materials. If SrO forms on the LSCF surface, $\mathrm{SrOH}_{2}$ can evaporate and flow outside the stack, thus resulting in novel $\mathrm{SrO}$ formation on the surface. Additionally, SrO interacts with $\mathrm{CrO}_{3} / \mathrm{Cr}(\mathrm{OH})_{2} \mathrm{O}_{2}$, forming $\mathrm{SrCrO}_{4}$. In the case of the volatile chromium species, they can be transported outside the stack, but are also able to interact with the perovskites used as a contacting or cathode material. The point of interaction depends on the underlying (electro)chemical reactions and the cathode material.

For the manganese oxide, no volatile species is likely to occur under SOFC operating conditions. Mn thus diffuses in the solid state and subsequently reacts under specific conditions within the electrolyte or at the electrolyte-anode interface, thus leading to secondary phase formation and subsequent delamination and cracking.

All interactions observed lead to the final conclusion that the materials within an SOFC stack should be chosen carefully with respect to (catalytic) activity and chemical stability (mostly contradictory), evaporation stability, long-term behavior and likelihood of interaction. Long-term operation in particular demonstrates the necessity for accelerated tests.

Ongoing model tests will clarify the reaction parameters which govern the severe materials interaction and the secondary phase formation.

\section{Acknowledgments}

The authors would like to thank X. Yin (IEK-2) for the FactSage calculations, Dr. E. Wessel (IEK-2) for the HAADF measurements, and U. de Haart and Prof. L. Blum (both IEK-3) for the stack operation.

\section{References}

1. L. Blum, L. G. J. de Haart, J. Malzbender, N. H. Menzler, J. Remmel and R. Steinberger-Wilckens, Journal of Power Sources, 241, 477 (2013).

2. $\quad$ R. Vaßen, N. Grünwald, D. Marcano, N. H. Menzler, R. Mücke, D. Sebold, Y. J. Sohn and O. Guillon, Surface and Coatings Technology, 291, 115 (2016).

3. N. Grünwald, N. H. Menzler, O. Guillon and R. Vaßen, Proc. 21st Hydorgen Energy Conference 2016, 1 (2016).

4. L. Blum, U. Packbier, I. C. Vinke and L. G. J. d. Haart, Fuel Cells, 13, 646 (2013).

5. N. H. Menzler, D. Sebold and Q. Fang, Journal of The Electrochemical Society, 162, F1275 (2015).

6. N. H. Menzler and P. Batfalsky, Fuel Cell Science and Engineering: Materiuals, Processes, Systems and Technology. Eds.: Detlef Stolten, Bernd Emonts, Vol. 1 (2012). Wiley-VCH, Weinheim, Germany, ISBN: 978-3-527-33012-6 
7. G. M. Rupp, H. Tellez, J. Druce, A. Limbeck, T. Ishihara, J. Kilner and J. Fleig, Journal of Materials Chemistry A, 3, 22759 (2015).

8. A. Beez, X. Yin, N. H. Menzler, R. Spatschek and M. Bram, Journal of the Electrochemical Society, submitted (2017). 